\title{
Biochemical Characterization, Kinetic Analysis and Molecular Modeling of Recombinant Vegetative Lipoxygenases from Soybean
}

\author{
George E. Sellhorn \\ Graduate Program in Molecular Plant Sciences, Washington State University \\ Pullman, Washington 99164-4234, USA \\ $\&$ \\ Viral Vaccine Program, Seattle Biomedical Research Institute, Seattle \\ Washington 98109, USA \\ BuHyun Youn \\ School of Molecular Biosciences, Washington State University \\ Pullman, Washington 99164-4234, USA \\ $\&$ \\ Department of Biological Sciences, Pusan National University \\ Pusan, Korea \\ Brian N. Webb \\ Department of Chemistry, Washington State University \\ Pullman, Washington 99164-4234, USA \\ Lisa M. Gloss \\ School of Molecular Biosciences, Washington State University \\ Pullman, Washington 99164-4234, USA \\ ChulHee Kang \& Howard D. Grimes (Corresponding author) \\ Graduate Program in Molecular Plant Sciences, Washington State University \\ \& School of Molecular Biosciences, Washington State University \\ Pullman, Washington 99164-4234, USA
}

Tel: (509)-335-6412Ｅ-mail: grimes@wsu.edu

This research was supported by the USDA, DOE, NSF and the Murdock Charitable Trust to HDG (DE-FG02-05ER15672)

\begin{abstract}
Plants in general have multiple lipoxygenase isoforms that exhibit distinct biochemical attributes and discrete cellular and sub-cellular localization patterns. This suggests that plant lipoxygenases are multifunctional and contribute to several physiological processes during growth and development. Soybeans have at least eight isoforms. Three that are localized to the seed (L-1; L-2; L-3) have been characterized extensively. The vegetative organs have five additional isoforms that are not well characterized. To better understand the potential cellular function of these vegetative lipoxygenases (VLXs), kinetic parameters and $\mathrm{pH}$ profiles with multiple substrates coupled with structural studies reveal differences within this family of enzymes. In addition, clear differences exist between VLX-A, -B \& -E and VLX-C \& -D in the exposed loops of the $\beta$-barrel, $\mathrm{O}_{2}$ cavity and entry site of the subcavity IIa. Therefore, based on distinct patterns of activity and structure, the existence of functional subgroups among the five VLX isoforms was confirmed.
\end{abstract}

Keywords: Vegetative lipoxygenase, Kinetics, Lipid metabolism, Molecular modeling 


\section{Introduction}

Lipoxygenases (LOXs, EC 1.13.11.12) are a family of non-heme iron containing dioxygenases that catalyze the regio- and stereo-specific oxidation of polyunsaturated lipids containing pentadiene systems and produce conjugated hydroperoxide products (Feussner \& Wasternack, 2002). LOXs are found in plants, animals, fungi and at least two bacterial species (Hamberg, 1998; Howe, Lightner, Browse \& Ryan, 1996; Rosahl, 1996; Samuelsson, 2000; Vidal-Mas, Busquets, \& Manresa, 2005; Koeduka, Kajiwara, \& Matsui, 2007). Free fatty acids (FFA) are the preferred substrates for LOXs; however, some LOX isoforms also utilize phospholipids and triglycerides (TG) as substrates (Brash, 1987; Fuller, Weichert, Fischer, Feussner, \& Grimes, 2001). In higher plants, LOXs prefer linoleic (18:2) and linolenic (18:3) acids as substrates and are characterized by their positional specificity for 18:2 oxygenation (Feussner \& Wasternack, 2002). In contrast, the primary endogenous substrate for mammalian LOXs is arachidonic acid (20:4) (Feussner \& Wasternack, 2002; Pande et al., 2004). So far the reported functions of plant LOX include: plant defense responses (Howe et al., 1996; Shah, 2005), stress responses (Ben-Hayim, Gueta-Dahan, Avsia-Kretchmer, Weichart, \& Feussner, 2001; Lee, Nioche, Hamberg, \& Raman, 2008; Gershenzon, 2007), plant microbe interactions (Porta \& Sosa, 2000), mobilization of TGs (Feussner, Kühn, \& Wasternack, 2001) and lipid metabolism (Feussner, et al., 2001; Gerhardt et al., Fischer et al., 2005).

Individual plant species typically have multiple LOX isoforms that differ in regards to spatial and temporal expression, $\mathrm{pH}$ optima, substrate specificity, product formation and enzyme kinetics (Feussner \& Wasternack, 2002). In soybean, which is the most thoroughly studied organism for LOX, eight lipoxygenases have been identified and cloned. The three seed LOXs, L-1, L-2, and L-3 are found in the cotyledons of non-germinated seeds, and have been extensively characterized (Feussner \&Wasternack, 2002; Oliw, 2002; Prigge, Faig, Doctor, Gaffney, \& Amzel, 1997). Five additional LOX isozymes (VLX-A,-B,-C,-D,-E) are found in the vegetative tissue of soybean. Enzyme kinetic studies have been completed for members of this large gene family and provide insight into their biological function. Steady-state kinetic analyses have been reported on the kinetic constants of soybean LOX-1 (Egmond, Brunori, \& Fasella, 1976; Knapp \& Klinman, 2003; Lagocki, Emken, Law, \& Kézdy, 1976; Smith \& Lands, 1972), LOX from olive fruit (Lorenzi, Maury, Casanova, \& Berti, 2007), dual positional specific maize LOX (Cho, Jang, Huon, Park, \& Han, 2007) and mini 9R-LOX (Andreou, Vanko, Bezakova, \& Feussner, 2008). Additional kinetic studies have been reported for mammalian 5-LOX, human 12\& 15-LOX and two fungal LOXs (Egan, Fluder, Gale, \& Brown, 1987; Pérez-Gilabert, Sánchez-Felipe, Morte, \& García-Carmona, 2005; Segraves \& Holman, 2003; Su, Sahlin, \& Oliw, 2000).

VLX-A,-B, \& -C accumulate in a unique cell layer termed the paraveinal mesophyll (PVM), a cell type that appears to be ideally positioned for metabolite transport. VLX-D preferentially accumulates in the palisade and spongy mesophyll parenchyma cells (Bunker et al., 1995; Franceschi \& Giaquinta, 1983a; Franceschi \& Giaquinta, 1983b; Franceschi \& Giaquinta, 1983c; Tranbarger, Franceschi, Hildebrand, \& Grimes, 1991). Initial biochemical studies from bacterial lysates of VLXs as MBP-fusion proteins indicated that all five enzymes are active against FFA. This suggests a role in lipid metabolism (Fuller et al., 2001; Fischer et al., 1999). In addition, VLX-C \& -D are active against TG, which further suggests a potential role in lipid catabolism (Fuller et al., 2001; Fischer et al., 1999).

In order to understand the potential roles of the specific isoforms in the metabolism of soybean lipids, a thorough investigation of substrate utilization and kinetic properties of five VLX isozymes was performed against 18:2, 18:3, 20:4 and TG, trilinolein. The observed clear differences in the biochemical characteristics between VLX-A, -B \&-E and VLC-C \& -D were further supported by the 3D structures of those five isozymes.

\section{Results}

\subsection{Expression/Purification}

VLX-A-E were expressed to high levels in E. coli for purification and characterization. M9 + Fe media was used for expression to reduce aggregation and further increased the specific activity of the VLXs. The optimal temperatures for expression are $15^{\circ} \mathrm{C}$ for VLX-C, $20^{\circ} \mathrm{C}$ for VLX-A, -B, \& -D and $25^{\circ} \mathrm{C}$ for VLX-E. The isozymes were purified by a combination of ammonium sulfate fractionation, and HPLC anion exchange and hydroxyapatite chromatography. The VLXs were purified to near homogeneity as verified by SDS-PAGE (Fig. S1). Purified VLX isozymes lost a significant portion of their activity within five days following purification and storage at $4^{\circ} \mathrm{C}$. Loss of activity was likely due to dissociation of the iron cofactor. Therefore, all the experiments reported in this article were performed within three days after purification. 


\section{$2.2 \mathrm{pH}$ activity profiles and substrate utilization}

The activities of the purified VLXs over the $\mathrm{pH}$ range of 4-9 were tested in order to determine the optimal $\mathrm{pH}$ for subsequent assays and to investigate the influence of $\mathrm{pH}$ on potential titratable groups. All five purified VLXs were characterized against the FFA, 18:2, 18:3, and 20:4 (Fig. 1). In addition, VLX-C \& -D were characterized against the TG, trilinolein (VLX-A, -B \& -E do not show activity against this substrate) (Fig. 1). VLX-A was active over a broad $\mathrm{pH}$ range against the three FFA but activity decreased sharply above $\mathrm{pH}$ 8.0. VLX-B shows broad activity against 18:2 but displayed decreased activity above physiological $\mathrm{pH}$ with 18:3 and 20:4. VLX-C and VLX-D had distinct $\mathrm{pH}$ optima in the physiological range for all three free fatty acids and were active over a broad $\mathrm{pH}$ range against trilinolein. VLX-E showed a preference for activity in the acidic range for all three free fatty acids. Additionally, there is a $\mathrm{pK}_{\mathrm{a}}$ between $\mathrm{pH}$ 6.5-7.0 for VLX-C \& -D. In general, the deprotonated state of the enzyme shows enhanced activity, but there is still significant activity with the protonated species. This suggests that the titrating group is not essential for catalysis and may be the carboxyl group of the fatty acid. The VLX isozymes were then tested for activity against three phospholipids, PS, PE and PC. For most VLX-phospholipid combinations, there was no activity observed. VLX-A \& -B showed low activity against PS with specific activities of 0.988 and $0.254 \mu \mathrm{Mol} \mathrm{O}{ }_{2} \mathrm{~min}^{-1} \mathrm{mg}^{-1}$. The low activities observed using phospholipid substrates suggest that these are not physiological substrates in vivo.

\subsection{Fatty acid backbone cleavage activity}

All five VLXs showed cleavage activity under the tested conditions (Table 1). The activities were quite low for most enzyme/substrate combinations tested when compared to the rate of oxygen uptake using the Polarographic assay and constitute only a small fraction of the product pool. Four combinations show cleavage activity above $20 \%$ of the rate of oxygen uptake; VLX-B against 18:2 \& 18:3 and VLX-E against 18:2 \& 20:4.

\subsection{Linolenic acid methyl ester activity}

The methyl ester of linolenic acid was tested as a substrate for the VLXs in order to determine if the uncharged fatty acid was able to bind to the enzyme and undergo peroxidation. This addressed the question of whether the charged carboxyl group of free fatty acids was required for substrate binding or stabilization during VLX catalysis. All five VLX isoforms were active against linolenic acid methyl ester (Fig. 2). VLX-A was the only isoform with higher activity against the methyl ester of linolenic acid compared to $18: 3$ and showed a 5 fold increase. VLX-B, -C, \& -D were less active against the methyl ester of linolenic acid relative to $18: 3$ with 1.5, 8.8 and 3.8 fold decreases observed, respectively. VLX-E had similar activities against both 18:3 and linolenic acid methyl ester.

\subsection{Enzyme kinetics}

The corresponding critical micelle concentration (CMC) values for 18:2, 18:3, and 20:4 were determined at $\mathrm{pH}$ 6.0, 6.5, 7.0 and 7.5 and range from $10-60 \mu \mathrm{M}$ (Table 2). In order to define the nature of the substrate under the reaction conditions, the CMC's were determined then kinetic analyses were completed against FFA, both above and below the CMC, for each substrate. Kinetic constants were also determined for VLX-C \& -D against the TG, trilinolein. All of these data were confirmed by at least two independent enzyme preparations. Due to the restriction of the substrate concentration limit for kinetic analysis of FFA, not all kinetic parameters could be determined below the CMC for some of the VLXs. For instance, at the limit of the substrate concentration for VLX-A, -B, \& -E, only a linear curve was established due to the inability to saturate the enzymes. Only $\mathrm{k}_{\mathrm{cat}} / \mathrm{K}_{\mathrm{m}}$, therefore, was analyzed for these three enzymes below the CMC which fall within the range of $10^{4}-10^{5} \mathrm{M}^{-1} \mathrm{x} \mathrm{s}^{-1}$ (Fig. 3a). VLX-A, -B \& -E all have similar $\mathrm{k}_{\text {cat }} / \mathrm{K}_{\mathrm{m}}$ values (split graph) for each substrate and are most efficient against 18:2 (Fig. 3a).

The ability of VLX-B to activate 18:2 for catalysis above $\mathrm{pH} 8.0$ may indicate that a difference exists within VLX-B that allows for abstraction of the hydride ion $\left(\mathrm{H}^{-}\right)$from 18:2, but not 18:3 or 20:4, prior to hydroperoxidation above $\mathrm{pH}$ 8.0. This could be a result of structural differences in the binding pocket of VLX-B compared to the other VLX isoforms. Alternatively, it could be a result of the fewer double bonds in 18:2 in combination with the structural difference observed between VLX-A and VLX-B. When comparing VLX-A and VLX-B the residues of the binding pockets are identical except at position 578. In VLX-A this residue is a methionine and in VLX-B this residue is a leucine. While neither of these residues can be ionized by changing the $\mathrm{pH}$, changes in the microenvironment of the binding pocket could be effected by $\mathrm{pH}$ and this in combination with the structural difference imposed by the methionine in VLX-A may contribute to the observed differences in catalytic activity above $\mathrm{pH} 8.0$ for VLX-B. 
Because of the lower $\mathrm{K}_{\mathrm{m}}$ values of VLX-C \& -D, a hyperbolic curve was established against the tested FFA substrates below the CMC. Both enzymes: display $\mathrm{k}_{\mathrm{cat}} / \mathrm{K}_{\mathrm{m}}$ values (Fig. 3a) in the range of $10^{6}-10^{7} \mathrm{M}^{-1} \mathrm{x} \mathrm{s}^{-1}$, have $\mathrm{k}_{\text {cat }}$ values (Fig. 3b) ranging from 39.4-244.0 $\mathrm{s}^{-1}$ and have $\mathrm{K}_{\mathrm{m}}$ values (Fig. 3c) in the low micromolar range. VLX-C \& -D are significantly more efficient enzymes than VLX-A, -B \& -E by one to two orders of magnitude (Fig. 3a) against all three substrates. VLX-C \& -D also have the highest turnover ( $\mathrm{k}_{\mathrm{cat}}$ ) (Fig. 3b) and $\mathrm{K}_{\mathrm{m}}$ (Fig. 3c) against 18:3 compared to other FFA. Interestingly, four of the five VLX isoforms are most efficient against 18:2 with VLX-C's preference for 20:4 being the exception (Fig. 3a).

Kinetic analysis of the VLXs above the CMC for the substrates tested was also performed. $\mathrm{K}_{\mathrm{cat}} / \mathrm{K}_{\mathrm{m}}$ values (Fig. 4a) for the five isoforms range from $10^{4}-10^{6} \mathrm{M}^{-1} \mathrm{x} \mathrm{s}^{-1}$, $\mathrm{k}_{\text {cat }}$ values (Fig. $4 \mathrm{~b}$ ) range from 2.8 to $376.3 \mathrm{~s}^{-1}$ and $\mathrm{K}_{\mathrm{m}}$ values (Fig. 4c) range from 18.4 to $335.1 \mu \mathrm{M}$. The evaluation of the $\mathrm{k}_{\mathrm{cat}} / \mathrm{K}_{\mathrm{m}}$ values for the VLXs against fatty acid micelles (FAM) show that VLX-C \& -D have much higher catalytic efficiency (again, one to two orders of magnitude) against FAM substrates compared to VLX-A, -B \& -E (Fig. 4a). VLX-C \& -D have distinctly higher $\mathrm{k}_{\text {cat }}$ values (split graph) against 18:3 micelles compared to other FAM (Fig. 4b) and the $\mathrm{K}_{\mathrm{m}}$ values are greater against 18:3 micelles compared with 18:2 and 20:4 micelles (Fig. 4c). Kinetic analysis of VLX-C \& -D against TG was completed as well. VLX-A, -B \& -E are not active against TGs (Fuller et al., 2001; Fischer et al., 1999). VLX-C \& -D displays $\mathrm{K}_{\mathrm{m}}$ values of $125.7 \mu \mathrm{M}$ and $202.2 \mu \mathrm{M}$, respectively, and very similar $\mathrm{k}_{\text {cat }} / \mathrm{K}_{\mathrm{m}}$ values of 3.4 $\mathrm{x} 10^{5} \mathrm{M}^{-1} \mathrm{x} \mathrm{s}^{-1}$ (VLX-C) and $2.9 \times 10^{5} \mathrm{M}^{-1} \mathrm{x} \mathrm{s}^{-1}$ (VLX-D). VLX-D has a slightly higher turnover number with a $\mathrm{k}_{\text {cat }}$ of $58.4 \mathrm{~s}^{-1}$ versus $42.2 \mathrm{~s}^{-1}$ for VLX-C.

For VLX-A, -B, \& -E there is no major difference between $\mathrm{k}_{\mathrm{cat}} / \mathrm{K}_{\mathrm{m}}$ against FFA compared with FAM. However, for VLX-C \& -D, it is clear that they are more efficient enzymes against FFA compared to FAM.

\subsection{Molecular modeling}

The corresponding 3-D structural models for VLX-A, -C \& -E were built to investigate the plausible explanation for the subtle difference in substrate specificities and product formation. This was possible due to a high level of sequence similarity among the five isozymes (Fig. 5) and availability of the crystal structures of VLX-B (2IUJ) and VLX-D (2IUK). None of the corresponding amino acid substitution produced any major high-energy Van der Waals contacts. Although there are a few areas of insertion or deletion in the exposed loop areas, especially in VLX-C \& -D, the overall structures were very similar to VLX-B and VLX-D (Fig. 6). In particular, the major insertion observed in VLX-C \& -D is located at the disordered loop on the N-terminal $\beta$-barrel facing the binding pocket, which connects $\beta 1$ and $\beta 2$ (Fig. 5, 6). The dotted line in red (Fig. 6) indicates the position of the disordered loop, including the insertion of VLX-A, -B \& -E. The dotted blue line indicates the disordered loop for in VLX-C \& -D.

\section{Discussion}

In the current study, we purified five VLX isozymes from soybean, analyzed their biochemical and kinetic properties, and built molecular models to further elucidate the structure-function relationships. In contrast to VLX-A, -B, \& -E, the strict $\mathrm{pH}$ dependence and much higher specific activity of VLX-C \& -D suggest that VLX-C \& -D are more likely to be involved in lipid metabolism. As shown in Figure 7, 8 and 9, the structural differences also clearly separate the five VLXs into two groups; VLX-A, -B \& -E comprising one group, and VLX-C \& -D constituting the other group.

Figure 7 shows an overlay of the residues dictating the shapes of the sub-cavity where productive substrate orientation and catalysis take place with the sub-cavity and residue number of VLX-B shown as a guide. The residues that are conserved among all five isozymes are shown in grey while the residues of heterogeneity are shown with colors. A few non-conserved residues appear important in relation to the observed differences in their enzyme activities as described above. Figure 8 displays the shape of the internal cavities, known as sub-cavity IIa, for all five VLX's. This is roughly composed of three branches: the extended pocket, entrance site and $\mathrm{O}_{2}$ cavity.

In every case, there is a barrier across the channel connecting the extended pocket with the remainder of the sub-cavity (Fig. 8). This may have important implication for their relative activities observed against linoleic acid and arachidonic acid, as noted previously (Youn, Sellhorn, Grimes, Mirchel, Gaffney, \& Kang, 2006). Among 11 residues that constitute the extended pocket, seven residues (F571, G715, I718, R721, T723, D761, and I765) are completely conserved and three others are conservative changes, however the residue A505 in VLX-B, which is also alanine in VLX-A, is substituted to a polar residue serine in VLX-C,-D \& -E. In particular, the extended pocket of the VLX-C is more extended compared to other four VLXs. 
Among the nine hydrophobic residues constituting this shortest $\mathrm{O}_{2}$ cavity branch, six residues are completely conserved among the five compared isozymes including the residues Q509, L560, I567 and L768. These residues were previously proposed to be the $\mathrm{O}_{2}$ blockers to the C-9 position of linoleic acid. Significantly all nine residues are identical between VLX-C \& -D. The remaining three residues of heterogeneity are I510, V561 and L578, among which the corresponding residues for V561 show a distinct pattern. VLX-A, -B \& -E contain a valine, at this position, which results in a broad area of contact between $\mathrm{O}_{2}$ cavity and fatty acid binding cavity, while the corresponding residue in VLX-C \& -D is an isoleucine resulting in a more narrow point of contact. The amide nitrogen of either valine or isoluecin residue, at this position, is in hydrogen bond position, with the backbone carbonyl oxygen of A556. This is completely conserved among five isozymes and was proposed to be a determinant for the stereochemistry and products by substrate orientation and flanking a pouch of $\mathrm{O}_{2}($ Oldham, Brash, \& Newcomer, 2005; Coffa et al., 2005).

As shown in Fig. 9, despite its less exposed character, the actual entrance site for the substrate of VLX-A, -B \& -E is slightly wider than that of VLX-C \& -D. Perhaps the most striking difference is A269, which is conserved among VLX-A, -B \&-E. The residue at the same position is substituted to a threonine in both VLX-C \& -D. The alanine residue allows for the entry site of the VLX-A, -B \& -E to be wider, while the same entry site are being restricted by the threonine in VLX-C \& -D (Fig. 9). In addition, the residue F25 of VLX-D, which is an isoleucine, I25, in VLX-B, forms a tighter hydrophobic cluster with the two residues in the entry site, F277 and Y280. Both of these residues are completely conserved among five VLXs. VLX-C also contains a phenylalanine, F27, in this position, while VLX-A and -E contain isoleucine. This hydrophobic cluster may further restrict the entry port for VLX-D \& -C, while VLX-A, -B \& -E, with an isoleucine in this position, would remain more accessible. While we observed that VLX-C displayed a substrate preference for 20:4, as observed for several mammalian LOXs, there is no readily identifiable structural basis for this preference.

In summary, based on the molecular models of VLX-A, -C \& -E described here, the crystal structures of VLX-B \& VLX-D (Youn et al., 2006) and previously reported biochemical data (Fuller et al., 2001; Fischer et al., 1999) there are distinct structure/function relationships among this family of enzymes. The soybean vegetative LOXs are all active, but act on different substrates with different kinetic parameters. This is consistent with other studies of mammalian and plant LOXs. (Andreou et al., 2008; Ben-Hayim et al., 2001; Brash, 1987; Cho et al., 2007; Egan et al., 1987; Fuller et al., 2001; Segraves \& Holman, 2003)

There is an ongoing debate concerning whether the fatty acid substrate binds with the methyl end or the carboxyl end buried in the lipoxygenase binding cavity. Experimental evidence supports both hypotheses (Egmond et al., 1976; Coffa et al., 2005). All five VLXs were able to utilize the methyl ester of linolenic acid as a substrate, which reflects that the VLX isoforms can bind an uncharged acyl chain and catalyze peroxidation. While these data do not provide detailed information about which end preferentially binds inside the binding pocket of the VLX isozymes, the decrease in activity of VLX-B-E against linolenic acid methyl ester indicates that the charged carboxyl group is involved in binding and stabilization of the enzyme-substrate complex. The specific activities of VLX-B-D against the methyl ester of 18:3 suggest an important role for the carboxyl group in substrate binding for these isoforms. These results also support our previous observation (Youn et al., 2006) that a basic residue, at the entrance of the binding pocket of VLX-B \& -D, enhances the interaction of ionized free fatty acids and the enzyme. In addition, the molecular models of VLX-A, -B \& -E and the crystal structures of VLX-B \& -D (Youn et al., 2006) show that the isozyme sub-cavities involved in substrate binding and $\mathrm{O}_{2}$ access are significantly different and are likely to contribute to the observed differences in positional specificity.

Evidence for the existence of C-C bond cleavage by the VLXs was shown previously and has focused on the production of the five carbon compounds pentenol and pentenone (Fischer, Grimes, \& Fall, 2003). This is of interest because the VLXs also may generate green leaf volatile (GLV) compounds which play important roles in biotic/abiotic stress, wounding, herbivore attack and exposure to ozone (Lee et al., 2008; Gershenzon, 2007). According to Salch et al., (1995), following $\beta$-scission of the fatty acid hydroperoxide, 13-OTA and the five-carbon products (pentenol and pentenone) were produced in addition to C-10 adducts. The specific product(s) generated by cleavage of 20:4 have not been determined thus reference to a specific $15 \mathrm{C}$ compound cannot be made. Fischer et al. (2003) state that the C5 radical reacts to give 1-penten-3-ol and 1-penten-3-one and discuss the major difference in the rate of oxygenation of fatty acids versus the production of $\mathrm{C} 5$ compounds with no mention of the potential adduct products. A possible explanation for this difference might be the result of the accumulation of $\mathrm{C} 10$ adducts. Our results analyzing C-C cleavage from the VLXs shows that there was a major difference in the rates of oxygen uptake versus the production of cleavage products. There was, however, a much greater fold difference between oxygen uptake and C-C cleavage from VLX-C \& -D compared with VLX-A, -B \& -E. More detailed analysis is required to elucidate the potential role of the VLXs in C-C cleavage 
and to determine the specific products produced by VLX-A, -B \& -E with 20:4 substrate. Recent studies have highlighted the ability of enzymes of the lipoxygenase pathway to contain multi-functional enzyme activity (Gao, Stumpe, Feussner, \& Kolomiets, 2008; Crechkin et al., 2008; Senger et al., 2005).

The results of our kinetic analysis of soybean VLXs indicate that VLX-A, -B \& -E are relatively inefficient enzymes against both FFA and FFM. However, VLX-C \& -D display characteristics that show the efficient utilization of those substrates under both substrate conditions as well as against the triglyceride trilinolein. The results from the experiments against FFA are better suited for interpreting the possible role of VLXs in lipid metabolism because the fatty acid concentration in the cell does not likely reach the level required for micelle formation. The kinetic parameters for the VLXs, in addition to the biochemical features of these enzymes, indicate that VLX-A, -B \& -E probably do not function as fatty acid metabolizing enzymes in soybean leaves. In contrast, VLX-C \& -D clearly display biochemical properties that support a role for these two isoforms in the metabolism of free fatty acids as well as triglycerides in soybean leaves. There are distinct patterns displayed in the kinetic parameters of VLX-C \& -D against 18:2 \& 18:3 which suggest a potential role of VLX-C \& -D in the remobilization of lipids in several cell types in the leaf. In addition to previous reports, these observations argue that these LOX isozymes may play important roles in nutrient mobilization in plants. The sub-cellular localization (Stephenson et al., 1998) and the ability of VLX-D to utilize TG provide a possible mechanism for remobilization of triacylglycerols stored in the palisade and spongy mesophyll cells. In addition, distinct structural features in the $\beta$-barrel domain observed in VLX-C \& -D may be critical for the ability of these two isoforms to bind triglycerides and catalyze hydroperoxidation. Further research is needed, however, to determine the biochemical mechanism of TG utilization by these two VLX isoforms.

Conclusion: In plants, the range of LOX products are involved in a wide array of downstream biochemical pathways (Feussner et al., 2001; Porta \& Rocha-Sosa, 2002). The aim of this study was to fully characterize the basic biochemical and structural features of VLX isozymes, which will help further elucidate their corresponding cellular functions and provide a tool for the future classification/identification of this family of enzymes. Data presented here support the hypothesis that recombinant VLXs display distinct structural and biochemical attributes between the groups and support a potential role for two isoforms in lipid metabolism in soybean leaves.

\section{Experimental procedures}

\subsection{Materials}

The reagents diphenylhexatriene (98\%), tetrahydrofuran (99.5\%), chloroamphenicol, linoleic, linolenic and arachidonic acids as well as phosphatidylserine (PS), phosphatidylethanolamine (PE) and phosphatidylcholine (PC) were from Sigma (St. Louis, MO). All enzyme substrates were a minimum of $99 \%$ purity. All substrates were prepared by diluting in $95 \%$ ethanol to $50 \mathrm{mM}$ with an $\mathrm{N}_{2}$ blanket and stored at $-20^{\circ} \mathrm{C}$ in sealed microcentrifuge tubes and all kinetic assays were completed from the same substrate stock for each replicate. Substrates were used within 3 months of preparation. The Oxygraph oxygen electrode was from Hansatech. All chemicals used were reagent grade.

\subsection{VLX expression}

Full length soybean VLX isozymes (Glycine max cv. Wye) have previously cloned into the pSBETa vector (Fuller et al., 2001) and harbored in BL21DE3PlysS E. coli cells (Stratagen, La Jolla, USA) for expression. Four liter cultures grown in $\mathrm{M} 9+\mathrm{Fe}$ medium $\left(42 \mathrm{mM} \mathrm{NaH}_{2} \mathrm{PO}_{4}, 22 \mathrm{mM} \mathrm{KH}_{2} \mathrm{PO}_{4}, 19 \mathrm{mM} \mathrm{NH}_{4} \mathrm{Cl}, 8.5 \mathrm{mM} \mathrm{NaCl}\right.$ plus $1 \mathrm{mM} \mathrm{MgSO}$. $0.2 \%$ glucose, $100 \mu \mathrm{M} \mathrm{Fe}\left(\mathrm{NH}_{4}\right)_{2}\left(\mathrm{SO}_{4}\right)_{2}$ with antibiotics were used for expression of the VLXs. Samples were harvested by centrifugation at $4000 \mathrm{xg}$ for 10 minutes. The supernatant was discarded and the pellets were flash frozen in liquid nitrogen and stored at $-80^{\circ} \mathrm{C}$ until use.

\subsection{Purification}

Following expression, frozen E.coli pellets were lysed using BugBuster $\AA$. Samples were centrifuged at 10,000xg for 30 minutes and the supernatant was fractionated with solid ammonium sulfate $(30-70 \%)$ at $4^{\circ} \mathrm{C}$. Pellets were resuspended in $20 \mathrm{mM}$ Tris $\mathrm{pH} 8.0$ and varied ionic strength $(\mathrm{NaCl})$ for the different isozymes. Samples were dialyzed overnight at $4^{\circ} \mathrm{C}$ against the same buffer to remove the ammonium sulfate. The purification of VLX-B and VLX-D has been described previously (Youn et al., 2006). Anion exchange chromatography using a MonoQ ${ }^{\text {TM }}$ GL10/100 column on a BioCad 700E preparative HPLC system was the initial chromatographic step. Desalted samples were loaded in low salt buffer as described above at $4 \mathrm{~mL} / \mathrm{min}$. Samples were eluted using 20 $\mathrm{mM}$ Tris $\mathrm{pH}$ 8.0, $1 \mathrm{M} \mathrm{NaCl}$ as follows: VLX-A was eluted using a 0.01-0.1 M linear gradient (4 mL), VLX-C was eluted with a 0.01-0.07 M linear gradient $(40 \mathrm{~mL})$ and VLX-E was eluted using a 0.001-0.25 $\mathrm{M}$ linear 
gradient $(4 \mathrm{~mL})$. VLX-containing fractions were dialyzed into $10 \mathrm{mM} \mathrm{NaH} \mathrm{PO}_{4}, \mathrm{pH} 6.8$ and $100 \mu \mathrm{M}$ $\mathrm{Fe}\left(\mathrm{NH}_{2}\right)\left(\mathrm{SO}_{4}\right)_{2}$ in preparation for hydroxyapatite chromatography using CHT-II resin (BioRad) and samples were loaded at $10 \mathrm{~mL} / \mathrm{min}$. VLX-A was eluted using a $0.01 \mathrm{M}-0.125 \mathrm{M}$ step gradient, VLX-C was eluted with a 0.01-0.095 M linear gradient (10 mL) and VLX-E was eluted using a 0.01 M - $0.2 \mathrm{M}$ step gradient. VLX-C \& -E samples were dialyzed into MonoQ loading buffer for re-chromatography on the anion exchange column with chloride as a counter ion (with the same $\mathrm{NaCl}$ concentration as for the initial MonoQ step). Elution during re-chromatography of VLX-C was at $4 \mathrm{~mL} / \mathrm{min}$ with at 0.01-0.075 linear gradient $(240 \mathrm{~mL})$. Re-chromatography of VLX-E utilized 0.001-0.1 M linear gradient ( $40 \mathrm{~mL}$ ). For VLX-A, pooled fractions were dialyzed into $20 \mathrm{mM}$ Tris $\mathrm{pH} 8.0,100 \mu \mathrm{M} \mathrm{Fe}\left(\mathrm{NH}_{2}\right)\left(\mathrm{SO}_{4}\right)_{2}$ and $5 \mathrm{mM}$ sodium acetate for re-chromatography on the MonoQ column using acetate as the counter ion. VLX-A was eluted using a linear gradient of 0.08-0.12 M (80 mL).

Purified VLXs were concentrated and buffered exchanged into $10 \mathrm{mM}$ MOPS pH 7.5, $25 \mathrm{mM} \mathrm{NaCl}$ and $100 \mu \mathrm{M}$ $\mathrm{Fe}\left(\mathrm{NH}_{2}\right)\left(\mathrm{SO}_{4}\right)_{2}$. Protein concentrations were determined by BCA assay. Purity of the VLX isozymes was confirmed by loading $1 \mu \mathrm{g}$ of protein per lane on a 12\% SDS-PAGE gel stained with Coomassie Brilliant Blue stain. All biochemical experiments were completed within 48 hours of final purification step.

\section{4 pH activity profiles}

The $\mathrm{pH}$ profiles were generated for VLX-A-E with purified protein in order to determine $\mathrm{pH}$ optima for kinetic experiments with 18:2, 18:3, and 20:4. Additionally, for VLX-C \& -D, pH profiles were generated for the TG, trilinolein. All assays were done at $25^{\circ} \mathrm{C}$ in $100 \mathrm{mM} \mathrm{NaH}_{2} \mathrm{PO}_{4}, \mathrm{pH} 4-9$ using the polarographic method with an Oxygraph oxygen electrode with the reaction cell temperature regulated with a circulating water bath.

\subsection{Phospholipid assays}

Activities of the VLXs against a variety of phospholipids, including PS, PE and PC, were conducted. Assays were performed at $25^{\circ} \mathrm{C}$ in $100 \mathrm{mM} \mathrm{NaH} \mathrm{PO}_{4}, \mathrm{pH} 7.0$ using the polarographic method. The substrate concentrations for all experiments were $0.5 \mathrm{mM}$.

\subsection{Linolenic acid methyl ester}

The activities of the VLXs against linolenic acid methyl ester were examined using the polarographic method at $25^{\circ} \mathrm{C}$ in $100 \mathrm{mM} \mathrm{NaH}_{2} \mathrm{PO}_{4}$ at the $\mathrm{pH}$ optimum for linolenic acid.

\subsection{Carbon-carbon $(C-C)$ backbone cleavage}

VLX fatty acid cleavage assays were done using 18:2, 18:3 and 20:4 on a Cary 50 Bio UV/Vis spectrophotometer (Varian) at the $\mathrm{pH}$ optimum for each isozyme for each substrate. The absorbance of $287 \mathrm{~nm}$ was recorded to measure the increase in accumulation of 13-oxotrienoic acid (13-OTA) for 18C fatty acids. The same absorbance was used to monitor the cleavage of 20:4 as well. A molar extinction coefficient of $28500 \mathrm{M}^{-1}$ $\mathrm{x} \mathrm{cm}^{-1}$ was used to calculate rates (Salch et al., 1995).

\subsection{Critical micelle concentration determination}

The critical micelle concentrations were determined at $\mathrm{pH}$ 6.0, 6.5, 7.0 and 7.5 using a fluorometric method (Chattopadhyay \& London, 1984) using a model ATF105 Titrating Differential/Ratio Spectrofluorometer (AVIV). The fluorescent dye, diphenylhexatriene (DPH) was excited at $358 \mathrm{~nm}$ and emission monitored at 430 $\mathrm{nm}$. DPH in tetrahydrofuran was prepared (Chattopadhyay \& London, 1984), and used at a final concentration of $0.25 \mu \mathrm{M}$. Briefly, $5 \mu \mathrm{l}$ of a lipid stock was added to a $2 \mathrm{~mL}$ reaction, so that there was a constant $0.25 \%(\mathrm{v} / \mathrm{v})$ EtOH concentration in each reaction. Micelle formation was assayed at lipid concentrations from 1 to $100 \mu \mathrm{M}$.

\subsection{Enzyme kinetic assays}

Reaction velocities were measured by the polarographic method (Pourplanche, Larreta-Garde, \& Thomas, 1991) using an Oxygraph oxygen electrode. The reaction vessel was maintained at $25^{\circ} \mathrm{C}$ by circulating water bath and stirring was controlled by the magnetic stirrer on the Oxygraph oxygen electrode. For enzyme kinetic assays, data were collected under two substrate concentration regimes-both above and below the CMC. A substrate concentration range of 1-500 mM was used above the CMC for all substrates. For assays below the CMC, the substrate concentrations were as follows; 1-20 mM for 18:2, 1-60 mM for 18:3 and 1-40 mM for 20:4. The concentration range of TG was from 1-500 mM for VLX-C \& -D TG kinetic assays. There is a linear relationship with regard to the enzyme concentration and enzymatic rate between 10-200 nM for VLX-A, -B \& -E for all three free fatty acid substrates. For VLX-C \& -D there is a linear relationship between 1-50 nM for the FFA as well as TG. 


\subsection{Molecular modeling}

Based on the distinct grouping of sequence similarity and the phylogenic tree between the five VLX isozymes, the corresponding models of VLX-A and VLX-E were built using the crystal structure of VLX-B (2IUJ in the Protein Data Bank) as a template, while the model of VLX-C was based on the structure of VLX-D (2IUK). Amino acid mutations were performed using the software O (Jones, Zou, Cowan, Kjeldgaard, 1991). After the initial models were built, an energy minimization was performed by CNS v1.2. (Brunger et al., 1998).The position and volume of subcavity IIa was calculated using the CASTp (Dundas et al., 2006) server, and all calculated pockets were visually inspected using the CASTp plug-in built for PyMOL. After indentifying which pockets were important for enzymatic activity, all further visualization was carried out in PyMOL.

\section{Acknowledgements}

This research was supported by the USDA, DOE, NSF and the Murdock Charitable Trust to HDG, (DE-FG02-05ER15672). The assistance of Brenda Droft in enzyme purification is gratefully acknowledged.

\section{References}

Andreou, A.Z., Vanko, M., Bezakova, L., \& Freussner, I. (2008). Properties of a mini 9R-lipoxygenase from Nostoc sp. PCC 7120 and its mutant forms. Phytochemistry, 69, 1832-37.

Ben-Hayim, G., Gueta-Dahan, Y., Avsia-Kretchmer, O., Weichart, H., \& Feussner, I. (2001). Preferential induction of a 9-lipoxygenase by salt in salt-tolerant cells of Citrus sinesis L. Osbeck. Planta, 212, 367-375.

Brash, A. (1987). Analysis of a specific oxygen reaction of soybean lipoxygenase-1 with fatty acids esterified in phospholipids. Biochemistry, 26, 55465-5471.

Brunger, A., Adams, P., Glore, G., DeLano, W., Gros, P., Grosse-Kunstleve, R., Jiang, J., Kuszewski, J., Nilges, M., Pannu, N., Read, R., Rice, L., Simonson, T., \& Warren, G. (1998). Crystallography \& NMR system: A new software suite for macromolecular structure determination. Acta Crystallogr D Biology Crystallogr, D54, 905-21.

Bunker, T.W., Koetje, D.S., Stephenson, L.C., Creelman, R.A., Mullet, J.E., \& Grimes, H.D. (1995). Sink limitations induces the expression of multiple soybean vegetative lipoxygenase mRNAs while the endogenous jasmonic acid level remains low. The Plant Cell, 7, 1319-1331.

Chattopadhyay, A., \& London, E. (1984). Fluorometric determination of critical micelle concentration avoiding interference from detergent charge. Analytical Biochem, 139, 408-412.

Cho, K., Jang, S., Huon, T., Park, S., \& Han, O. (2007). Biochemical characterization of the dual positional specific maize lipoxygenase and the dependence of lagging and initial burst phenomenon on $\mathrm{pH}$, substrate, and detergent during pre-steady state kinetics. Journal of Biochemistry and Molecular Biology, 40, 100-06.

Coffa, G., Imber, A.N., Maguire, B.C., Laxmikanthan, G., Schneider, C., Gaffney, B.J., \& Brash, A.R. (2005). On the relationships of substrate orientation, hydrogen abstraction, and product stereochemistry in single and double dioxygenations by soybean lipoxygenase-1 and its Ala54Gly mutant. Journal of Biological Chemistry, 280, 38756-66.

Dundas, J., Ouyang, Z., Tseng, J., Binkowski, A., Turpaz, Y., and Liang, J. (2006). CASTp: computed atlas of surface topography of proteins with structural and topographical mapping of functionally annotated residues. Nucl. Acids Res., 34:W116-W118.

Egan, R.W., Fluder, E.J., Gale, P.H., \& Brown, S.A. (1987). Molecular modeling and enzyme kinetics indicate a novel mechanism for mammalian 5-lipoxygenase. Advances in Prostaglandin, Thromboxane and Leukot Research, 17A, 69-74.

Egmond, M.R., Brunori, M., \& Fasella, P.M. (1976). The steady-state kinetics of the oxygenation of linoleic acid catalyzed by soybean lipoxygenase. European Journal of Biochemistry, 251, 93-100.

Feussner, I., Kühn, H., Wasternack, C. (2001). Lipoxygenase-dependent degradation of storage lipids. TRENDS in Plant Sciences, 6, 268-273.

Feussner, I., \& Wasternack, C. (2002). The lipoxygenase pathway. Annual Review of Plant Biology, 53, 275-297. Fischer, A.M., Dubbs, W.E., Baker, R.A., Fuller, M.A., Stephenson, L.C., \& Grimes, H.D. (1999). Protein dynamics, activity and cellular localization of soybeans lipoxygenases indicate distinct functional roles for individual isoforms. The Plant Journal, 19, 543-554. 
Fischer, A.J., Grimes, H.D., \& Fall, R. (2003). The biochemical origin of pentenol emissions from wounded leaves. Phytochemistry, 62, 159-63.

Franceschi, V.R., \& Giaquinta, R.T. (1983a). The paraveinal mesophyll of soybean leaves in relation to assimilate transfer and compartmentation. I. Ultrastructural and histochemistry during vegetative development. Planta, 157, 411-21.

Franceschi, V.R., \& Giaquinta, R.T. (1983b). The paraveinal mesophyll of soybean leaves in relation to assimilate transfer and compartmentations. II. Structural, metabolic and compartmental changes during reproductive growth. Planta, 157, 422-31.

Franceschi, V.R., \& Giaquinta, R.T. (1983c). Specialized cellular arrangements in legume leaves in relation to assimilate transport and compartmentation. Comparison of the paraveinal mesophyll. Planta, 159, 415-22.

Fuller, M.A., Weichert, H. Fischer, A.M., Feussner, I., \& Grimes, H.D. (2001). Activity of soybean lipoxygenases against esterified fatty acids indicates functional specificity. Archives of Biochemistry and Biophysics, 388, 146-54.

Gao, X., Stumpe, M., Feussner, I., \& Kolomiets, M. (2008). A novel plastidial lipoxygenase of Maize (Zea Mays) ZmLOX6 encodes for a fatty acid hydroperoxide lyase and is uniquely regulated by phytohormones and pathogen infection. Planta, 491-503.

Gerhardt, B., Fischer, K., Balkenholhl, T.J., Pohnert, G., Kühn, H., Wasternack, C., \& Feussner, I. (2005). Lipoxygenase-mediated metabolism of storage lipids in germinating sunflower cotyledons and B-oxidation of (9Z, 11E, 13S)-13hydroxy-octadeca-9, 11-dienoic acid by the cotyledonary glyoxysomes. Planta, 220, 919-30.

Gersheonzon, J. (2007). Plant volatiles carry both public and private messages. PNAS USA, 104, 5257-58.

Grechkin, A.N., Mukhtarova, L.S., Latypova, L.R., Gogolev, Y., Toporkova, Y.Y., \& Hamberg, M. (2008). Tomato CYP74C3 is a multifunctional enzyme not only synthesizing allene oxide but also catalyzing its hydrolysis and cyclization. Chembiochem, 9, 2498-2505.

Grimes, H.D., Tranbarger, T.J., \&Franceschi, V.R. (1993). Expression and accumulation patterns of nitrogen-responsive lipoxygenase in soybean. Plant Physiology, 103, 457-466.

Hamberg, M. (1998). A pathway for biosynthesis of divinyl ether fatty acids in green leaves. Lipids, 33, 1061-1071.

Howe, G.A., Lightner, J., Browse, J., \& Ryan, C.A. (1996). An octadecanoid pathway mutant (JL5) of tomato is compromised in signaling for defense against insect attack. The Plant Cell, 8, 2067-77.

Jones, T.A., Zou, J.Y., Cowan, S.W., Kjeldgaard, M. (1991). Improved methods for building protein models in electron density maps and the location of errors in these models. Acta Crystallogr A, 47, 110-19.

Knapp, M.J., \& Klinman, J.P. (2003). Kinetic studies of oxygen reactivity in soybean lipoxygenase-1. Biochemistry, 79, 629-36.

Koeduka, T., Kajiwara, T., \& Matsui, K. (2007). Cloning of lipoxygenase genes from cyanobacterium, Nostoc punctiforme, and its expression in Escherichia coli. Current Microbiology, 54, 315-9.

Lagocki, J.W., Emken, E.A., Law, J.H., \& Kezdy, J.F. (1976). Kinetics analysis of the action of soybean lipoxygenase on linoleic acid. Journal of Biological Chemistry, 251, 6001-06.

Lee, D.S., Nioche, P., Hamberg, M., \& Raman, C.S. (2008). Structural insights into the evolutionary paths of oxylipin biosynthetic enzymes. Nature, 455, 363-70.

Lorenzi, V., Maury, J., Casanova, J., \& Berti, L. (2007). Purification, product characterization and kinetic properties of lipoxygenase from olive fruit. Plant Physiology and Biochemistry, 44, 450-54.

Oldham, M., Brash, A., Newcomer, M. (2005). Insights from the X-ray crystal structure of coral 8R-lipoxygenases: calcium activation via A C2-like domain and a structural basis of product chirality. Journal of Biological Chemistry, 280, 39545-52.

Oliw, E.W. (2002). Plant and fungal lipoxygenases. Prostaglandins and Other Lipid Mediators, 68-69, 313-23.

Pande, A.H., Moe, D., Nemec, K.N., Qin, S., Tan, S., \& Tatulian, S.A. (2004). Modulation of human 5-lipoxygenase activity by membrane lipids. Biochemistry, 43, 14653-66. 
Pérez-Gilabert, M., Sánchez-Felipe, I., Morte, A., and \& García-Carmona, F. (2005). Kinetic properties of lipoxygenae from desert truffle (Terfezia claveryi Chatin) asocarps: Effect of inhibitors and activators. Journal of Agriculture and Food Chemistry, 53, 6140-45.

Porta, H., \& Rocha-Sosa, M. (2000). A Phaseolus vulgaris lipoxygenase gene expressed in nodules and in Rhizobium tropici inoculated roots. Biochem Biophys Acta, 1517, 139-42.

Porta, H., \& Rocha-Sosa, M. (2002). Plant lipoxygenases: Physiological and molecular features. Plant Physiology, 130, 15-21.

Pourplanche, C., Larreta-Garde, V., \& Thomas, D. (1991). Comparison of polarographic and chemical measurements of oxygen uptake in complex media: the example of lipoxygenase reaction. Analytical Biochemistry, 198, 160-164.

Prigge, J.C., Faig, M., Doctor, K.S., Gaffney, B.J., \& Amzel, L.M. (1997). Structure and mechanism of lipoxygenase. Biochimie, 79, 629-36.

Rosahl, S. (1996). Lipoxygenases in plants-Their role in development and stress response. Naturforsch, 51, 123-38.

Salch, Y.P., Gove, M.J., Takamura, H., \& Gardner, H.W. (1995). Characterization of a C-5, 13-cleaving enzyme of 13(S)-hydroperoxide linolenic acid by soybean seed. Plant Physiology, 108, 1211-18.

Samuelsson, B. (2000). The discovery of the leukotrienes. American Journal of Respiratory and Critical Care Medicine, 161, S2-S6.

Segraves, E.N., \& Holman, T. (2003). Kinetic investigations of the rate-limiting step in human 12 and 15-lipoxygenase. Biochemistry, 42, 5236-43.

Senger, T., Wichard, T., Kunze, S., Göbel, C., Lerchl, J., Pohnert, G., \& Feussner, I. (2005). A multifunctional lipoxygenase with fatty acid hydroperoxide cleaving activity from the moss Physcomitrella patens. Journal of Biological Chemistry, 280, 7588-96.

Shah, J. (2005). Lipids, lipases and lipid-modifying enzymes in plant disease resistance. Annual Review of Phytopathology, 43, 229-60.

Smith, W.L., \& Lands, W.E.M. (1972). Oxygenation of unsaturated fatty acids by soybean lipoxygenase. Journal of Biological Chemistry, 247, 1038-47.

Stephenson, L.C., Bunker, T.J., Dubbs, W.E., \& Grimes, H.D. (1998). Specific soybean lipoxygenases localize to discrete subcellular compartments and their mRNAs are differentially regulated by source-sink status. Plant Physiology, 116, 923-33.

Su, C., Sahlin, M., \& Oliw, E.H. (2000). Kinetics of manganese lipoxygenase with a catalytic mononuclear redox center. Journal of Biological Chemistry, 275, 18830-35.

Tranbarger, T.J., Franceschi, V.R., Hildebrand, D.F., \& Grimes, H.D. (1991). The soybean 94 kilodalton vegetative storage protein is a lipoxygenase that is localized in the paraveinal mesophyll cell vacuoles. The Plant Cell, 3, 973-87.

Vidal-Mas, J., Busquets, M., \& Manresa, A. (2005). Cloning and expression of a lipoxygenase from Psedudomonas aeruginosa 42A2. Antonie van Leeuwenhoek, 87, 245-51.

Youn, B., Sellhorn, G.E., Grimes, H.D., Mirchel, R.J., Gaffney, B.J., \& Kang, C. (2006). Crystal structures of soybean vegetative lipoxygenase VLX-B and VLX-D. Proteins, 65, 1008-20. 
Table 1. Cleavage rate of C18 (18:2 \& 18:3) and C20 (20:4) backbones of VLX-A-E. U= $\mu$ mol 13-OTA min ${ }^{-1}$. Rates are expressed in U/mg of 13-oxotrienoioc for 18:2 \& 18:3 (15C oxo-acid for 20:4). a) percent rate of oxygenation of 18:2. b) percent rate of oxygenation of 18:3. c) percent rate of oxygenation of 20:4.

Fatty acid cleaving activity of VLXs.

\begin{tabular}{lcccccc}
\hline & $18: 2$ & $\mathrm{a}$ & $18: 3$ & $\mathrm{~b}$ & $20: 4$ & $\mathrm{c}$ \\
\hline VLX-A & 0.314 & 17.3 & 0.169 & 7.0 & 0.211 & 10.7 \\
VLX-B & 0.426 & 26.2 & 0.160 & 6.90 & 0.240 & 15.0 \\
VLX-C & 5.62 & 2.97 & 34.3 & 16.6 & 4.39 & 2.96 \\
VLX-D & 3.09 & 9.47 & 19.8 & 22.0 & 4.51 & 6.89 \\
VLX-E & 0.566 & 36.1 & 0.291 & 8.24 & 0.439 & 34.5 \\
\hline
\end{tabular}

Table 2. Critical micelle determination for free fatty acids. A fluorometric method was utilized to determine the CMCs for 18:2, 18:3 \& 20:4 in $100 \mathrm{mM} \mathrm{Na}_{2} \mathrm{PO}_{4}$ at $\mathrm{pH} 6.0,6.5,7.0$ \& 7.5.

Critical Micelle Concentration

$(\mu \mathrm{M})$

\begin{tabular}{llll}
\hline & $18: 2$ & $18: 3$ & $20: 4$ \\
\hline pH 6.0 & 15 & 20 & 10 \\
pH 6.5 & 60 & 30 & 10 \\
pH 7.0 & 20 & 60 & 40 \\
pH 7.5 & 20 & 60 & 40 \\
\hline
\end{tabular}




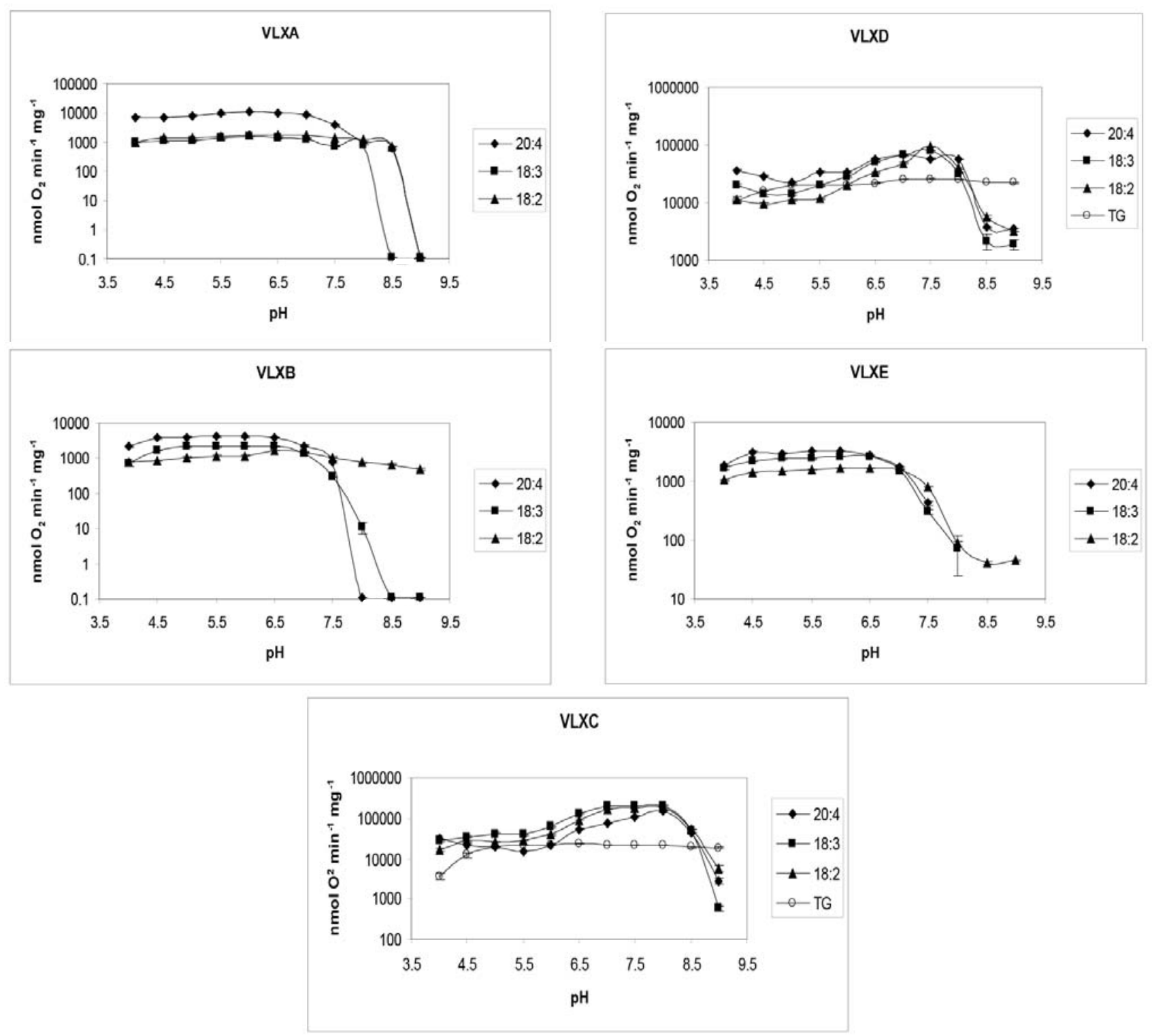

Figure 1. $\mathrm{pH}$ curves (log-log) of VLX-A-E. Results from activity assays for VLX-A-E against FFA and trilinolein (VLX-C \& VLX-D) in $100 \mathrm{mM} \mathrm{Na}_{2} \mathrm{HPO}_{4}$ from $\mathrm{pH}$ 4-9. Rates are expressed as nmol of $\mathrm{O}_{2} \mathrm{~min}^{-1} \mathrm{mg}^{-1}$ (specific activity). The substrate concentration was $0.5 \mathrm{mM}$ for all assays. Linoleic acid (18:2), linolenic acid (18:3), arachidonic acid (20:4), trilinolein (TG). 


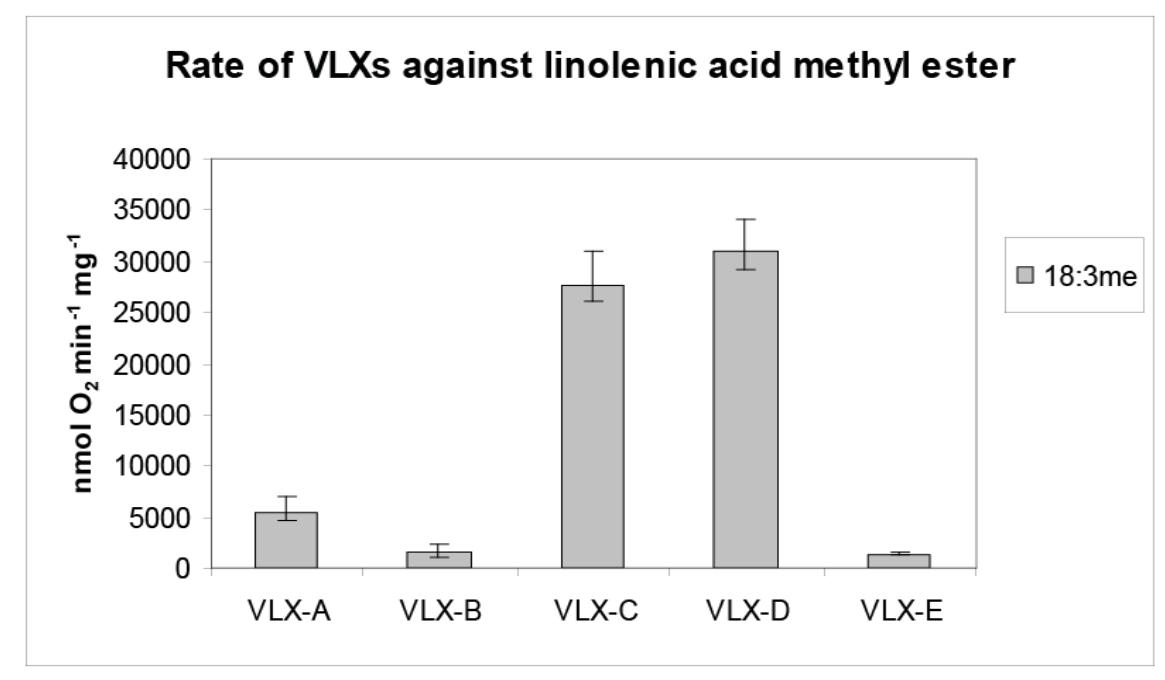

Figure 2. Relative activities of VLX-A-E against linolenic acid methyl ester (18:3me). Specific activities of VLX-A,-B,-C,-D \& -E against linolenic acid methyl ester were determined in $100 \mathrm{mM} \mathrm{Na}_{2} \mathrm{HPO}_{4}$ at the $\mathrm{pH}$ optimum determined for each isozyme against linolenic acid: VLX-A, pH 6.0, VLX-B, pH 6.5, VLX-C, pH 8.0, VLX-D, pH 7.5, VLX-E, pH 6.0. The substrate concentration was $0.5 \mathrm{mM}$ for all assays. Rates are expressed as nmol of $\mathrm{O}_{2} \mathrm{~min}^{-1} \mathrm{mg}^{-1}$.

(A)

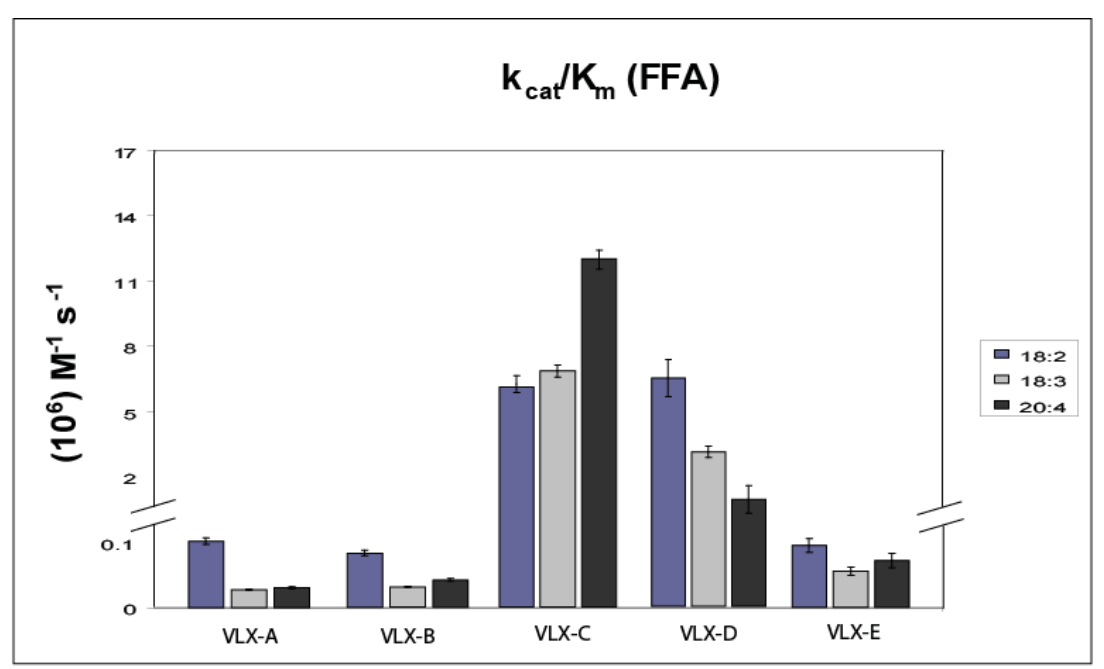


(B)

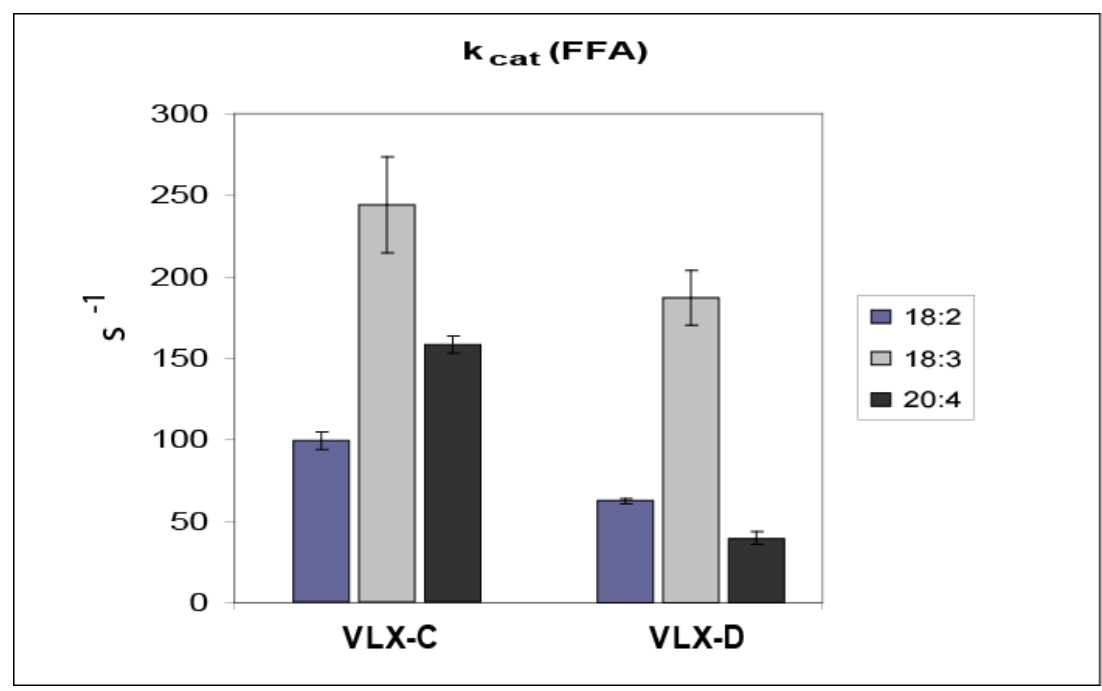

(C)

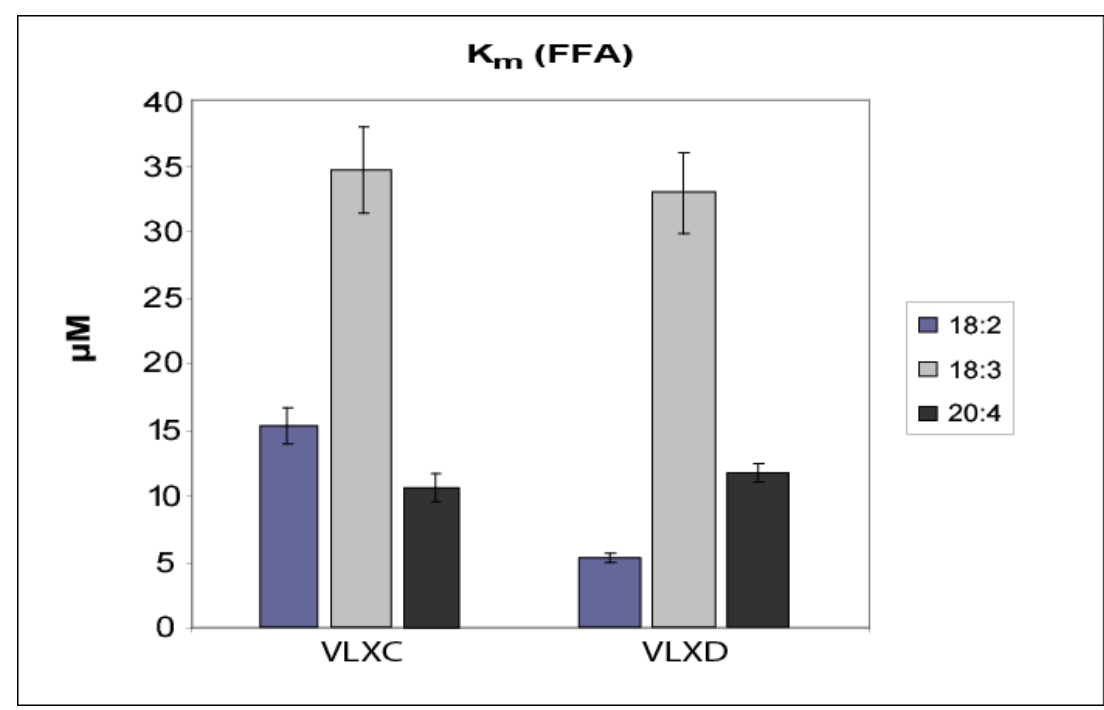

Figure 3. Analysis of VLX kinetic parameters for free fatty acids. A) Comparison of $\mathrm{k}_{\mathrm{cat}} / \mathrm{K}_{\mathrm{m}}$ for the five isoforms (split graph). B) Comparison of $\mathrm{k}_{\mathrm{cat}}$ values for VLX-C and VLX-D. C) Comparison of $\mathrm{K}_{\mathrm{m}}$ values for VLX-C \& VLX-D. All assays done in $100 \mathrm{mM} \mathrm{Na}_{2} \mathrm{HPO}_{4}$ at the $\mathrm{pH}$ optimum for each substrate. 
(A)

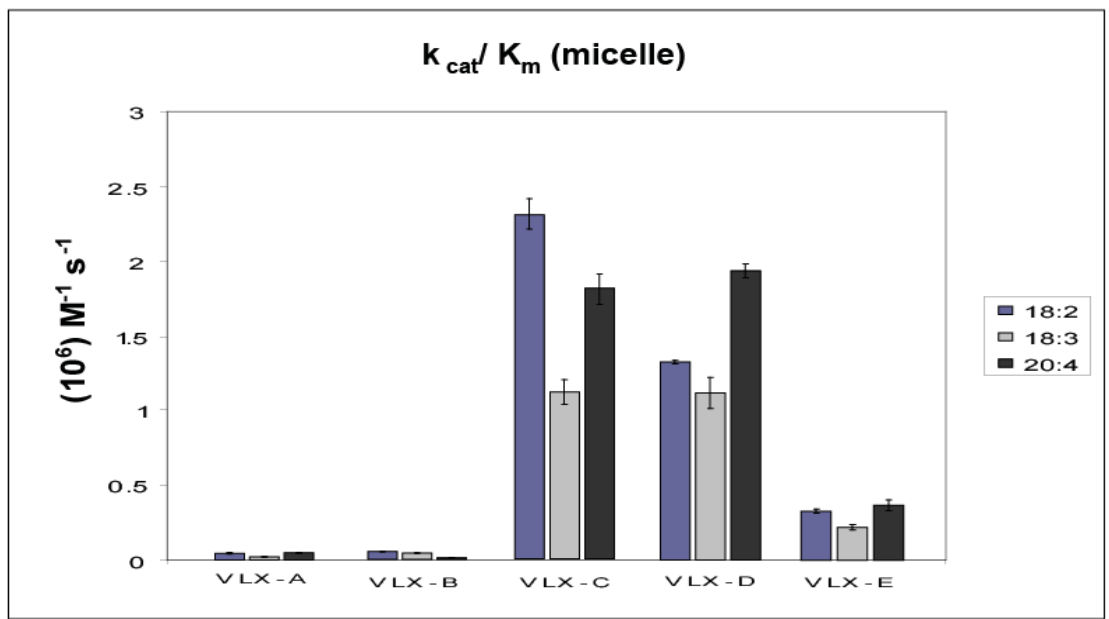

(B)

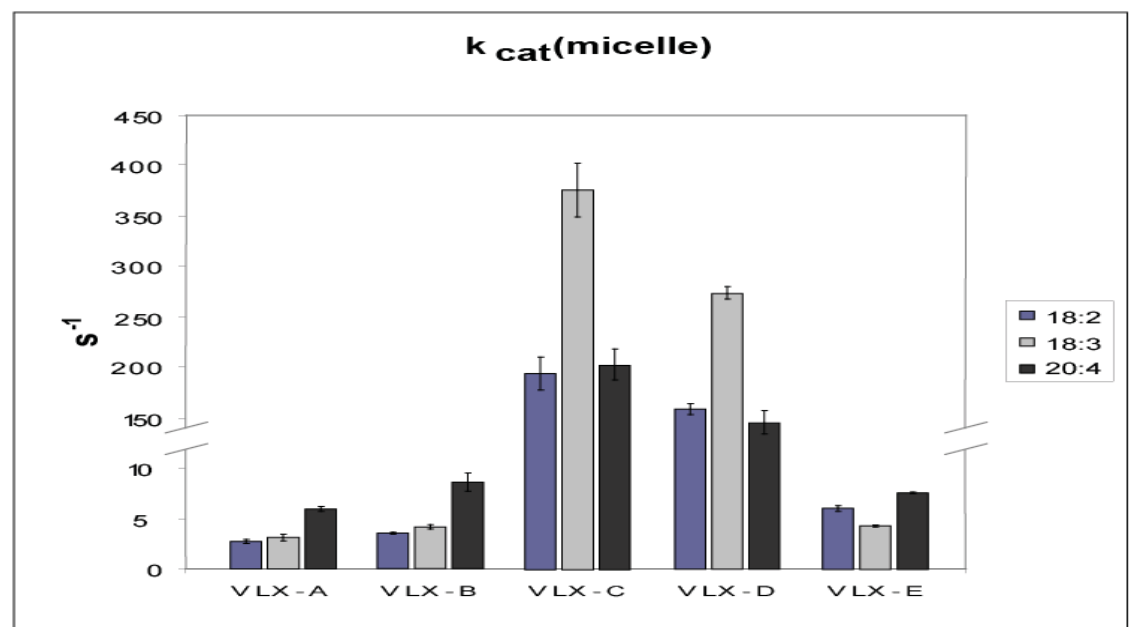

(C)

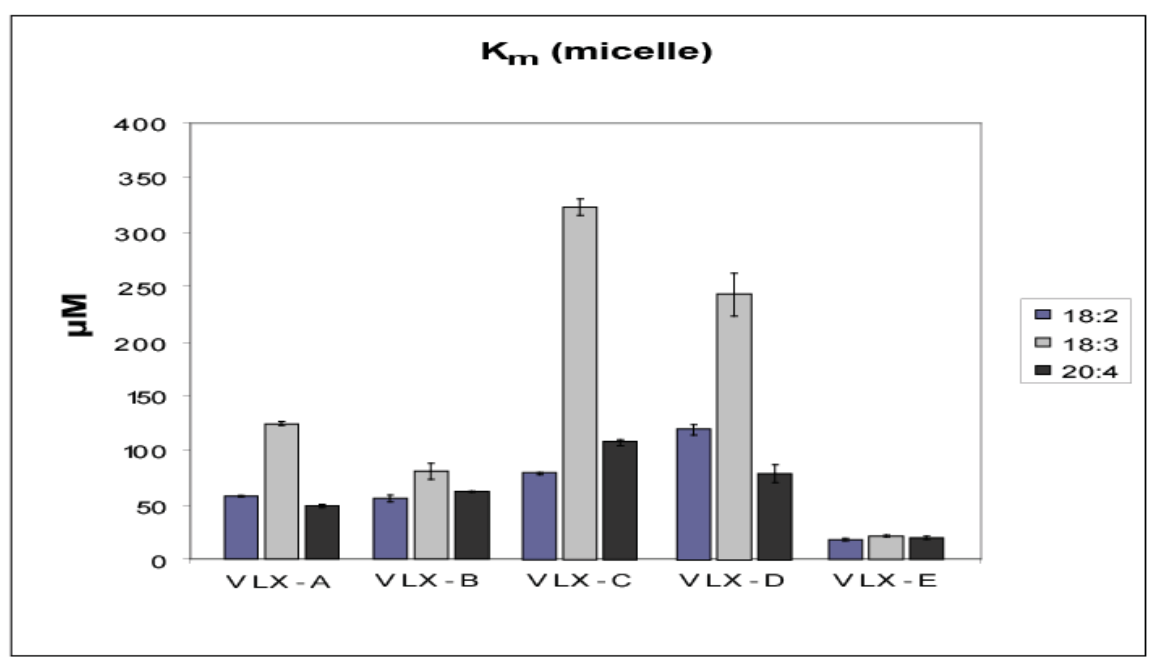

Figure 4. Analysis of VLX kinetic parameters for fatty acid micelles. A) Comparison of $\mathrm{k}_{\text {cat }} / \mathrm{K}_{\mathrm{m}}$ values for VLX-A-E (split graph). B) Comparison of $\mathrm{k}_{\mathrm{cat}}$ values for VLX-A-E (split graph). C) Comparison of $\mathrm{K}_{\mathrm{m}}$ values for VLX-A-E. All assays done in $100 \mathrm{mM} \mathrm{Na}_{2} \mathrm{HPO}_{4}$ at the $\mathrm{pH}$ optimum for each substrate. 


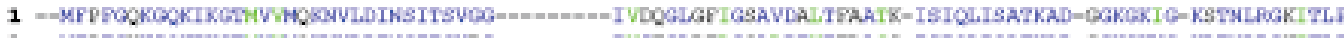
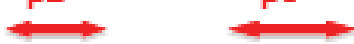

VLXB

VLXC

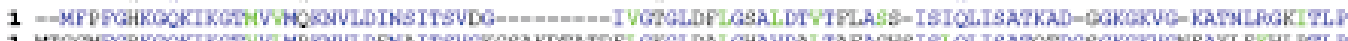

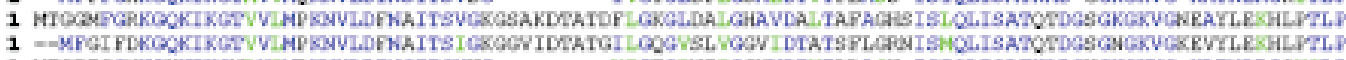

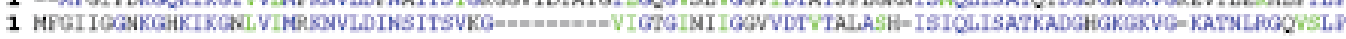

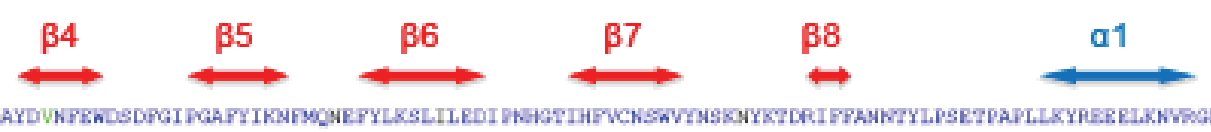

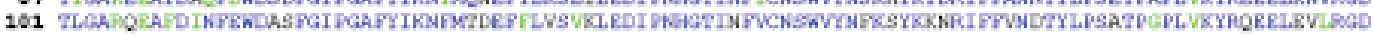

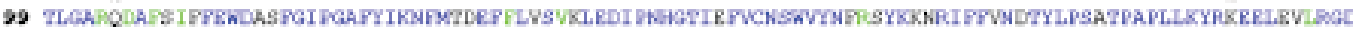

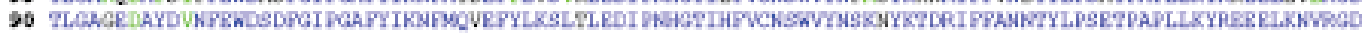

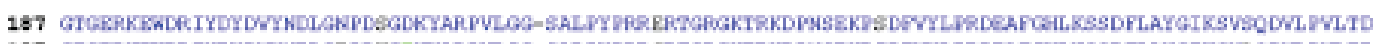

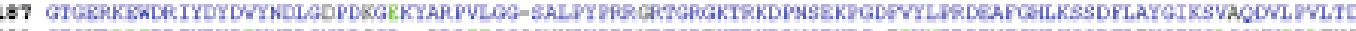

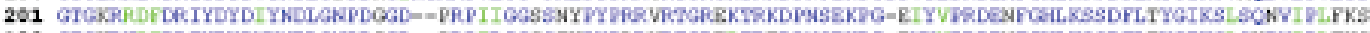

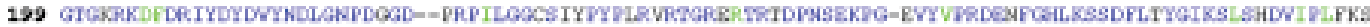

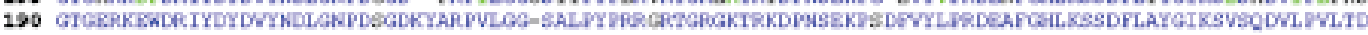
$\Rightarrow$
$\stackrel{\alpha 3}{\longrightarrow} \beta 11 \stackrel{\alpha 4}{\longrightarrow}$
$\beta 12$

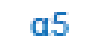
* $\mathrm{\beta} 13$

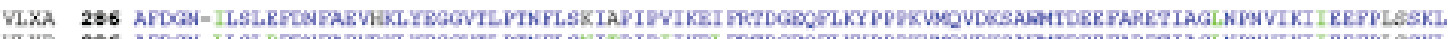

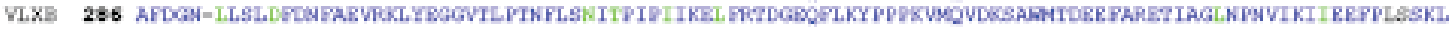

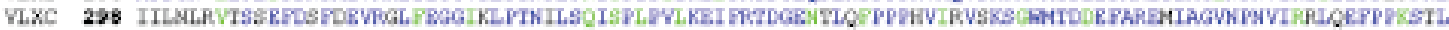

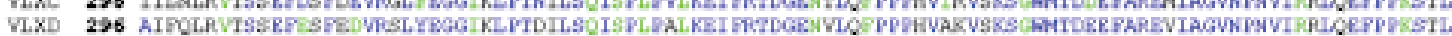

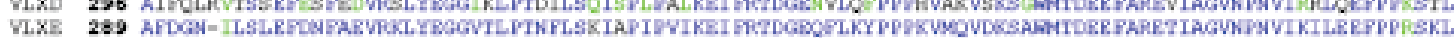

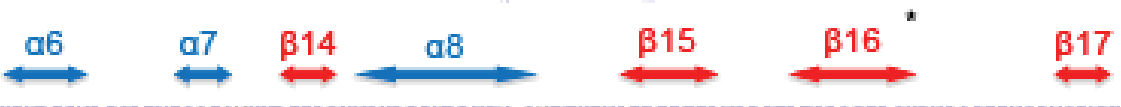

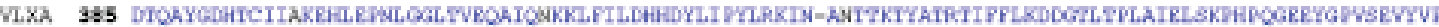
TLX $\mathrm{X}$ :

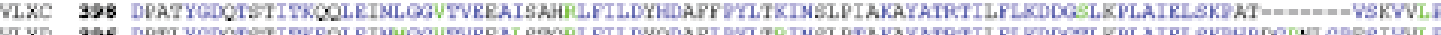

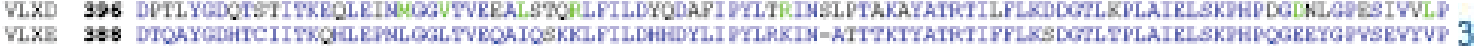

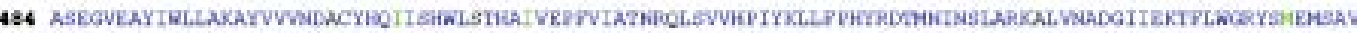

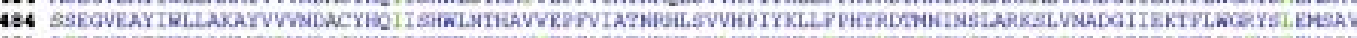

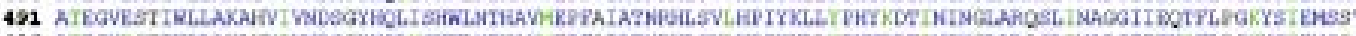

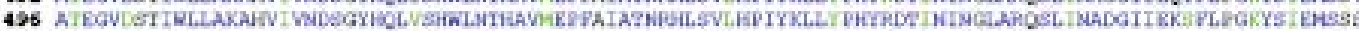

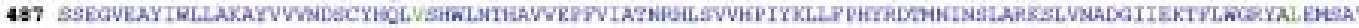
$\Rightarrow \quad$ a14 $\dot{\beta}_{\omega} 18^{* \prime} \dot{\beta} 19$
a15
a16
a17
at as

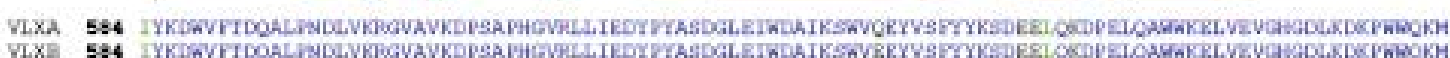



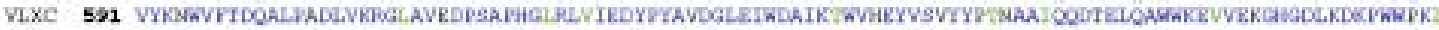

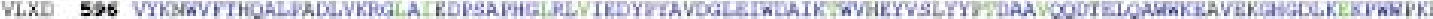

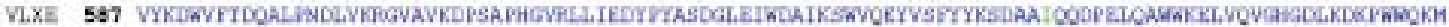



Figure 5. Multiple amino acid sequence alignment for VLX-A, VLX-B, VLX-C, VLX-D \& VLX-E: The residues that are identical among five isozymes are depicted with blue color, the conserved residues are depicted with green and non-conserved residues are with black. The specific residues of the various cavities discussed in the text are marked with an asterisk $(*)$. 


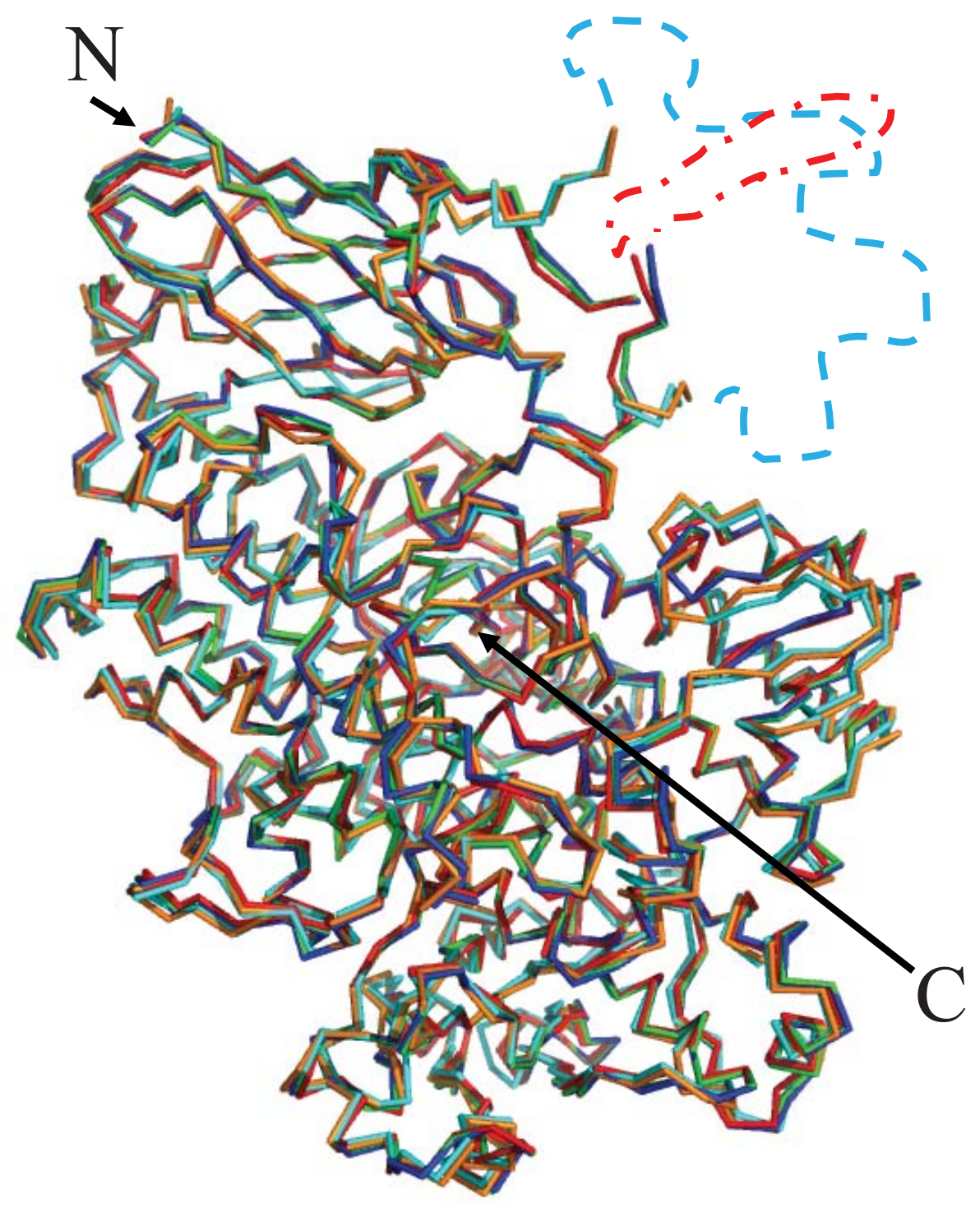

Figure 6. Superimposed views of $\alpha$ C positions of VLXs. VLX-A, VLX-B, VLX-C, VLX-D \& VLX-E are depicted in green, red, orange, cyan and blue respectively. Dotted red line indicates VLX-A, -B \& -E loops and the dotted blue line indicates the VLX-C\& -D loops. Black arrows indicate N \& C termini. This figure was generated with the program PyMOL (v 0.99). 


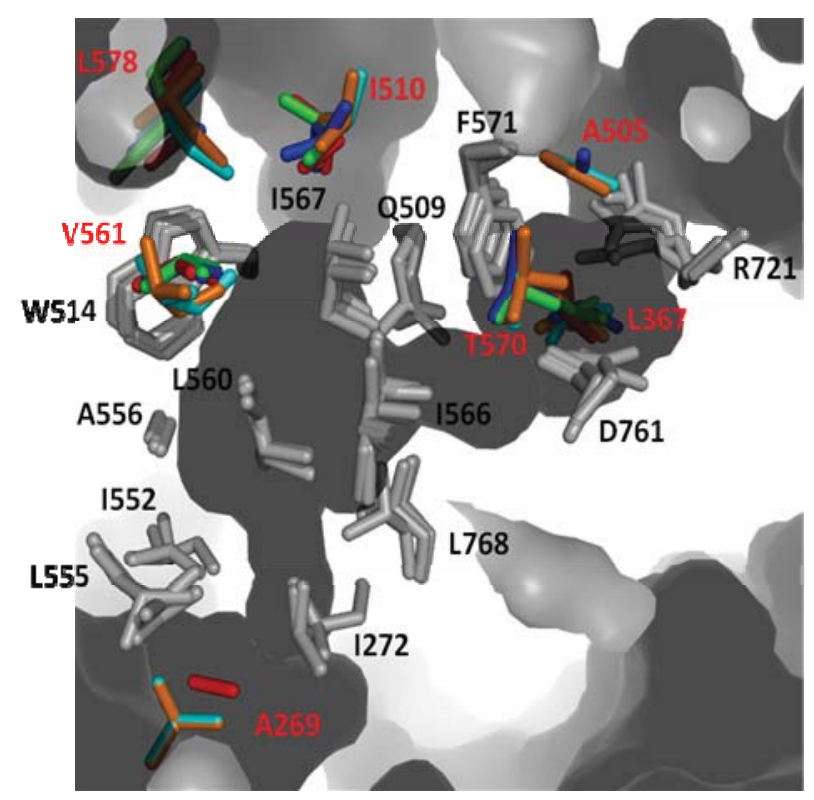

Figure 7. The residues constituting the cavity IIa: conserved residues are represented by gray (with black residue number) and the non-conserved residues were represented by colors (with red residue number). VLX-A, VLX-B, VLX-C, VLX-D and VLX-E are depicted in green, red, orange, cyan and blue respectively. For the clarity of this discussion, the numbering system of VLX-B is being used.

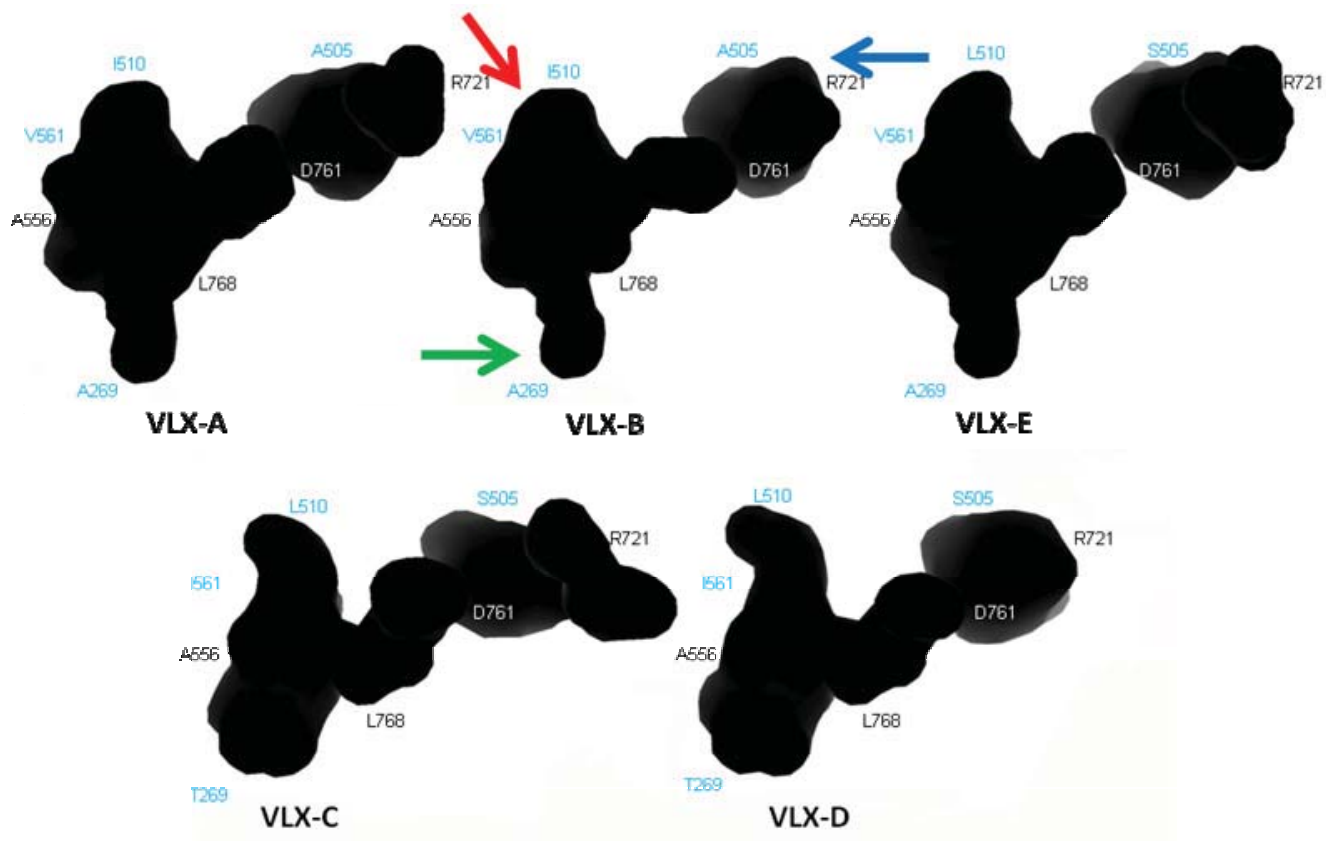

Figure 8. The shape of subcavity IIa of VLX-A, VLX-B, VLX-C, VLX-D and VLX-E: The $\mathrm{O}_{2}$ cavity (red arrow), extended cavity (blue arrow) and entrance site (green arrow) are indicated. After indentifying which pockets were important for enzymatic activity, all further visualization was carried out in CASTp (Dundas et al., 2006) and PyMOL. The volume of subcavity IIa for VLXA, VLXB, and VLXE were calculated to be 354.2, 332.0 , and $354.6 \AA^{3}$, respectively, with the volumes of the extended pockets calculated at 249.2, 156.6, and $305.3 \AA^{3}$. The volume of subcavity IIa for VLXC and VLXD were found to be 306.3 and $286.6 \AA^{3}$, respectively, while the volumes of the extended pockets were 238.5 and $179.5 \AA^{3}$. For the clarity of this discussion, the numbering system of VLX-B is being used. This figure was generated with the program PyMOL (v 0.99). 


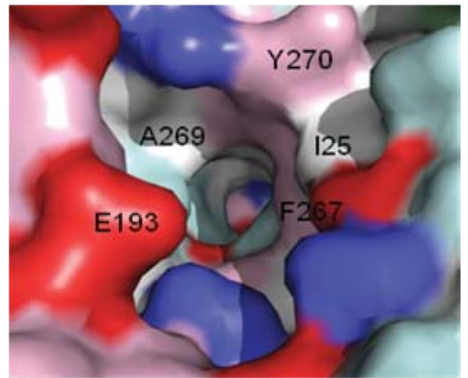

VLX-A

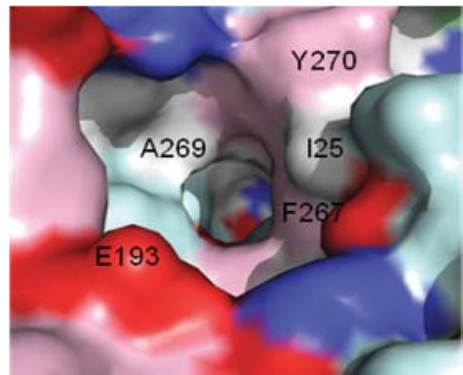

VLX-B

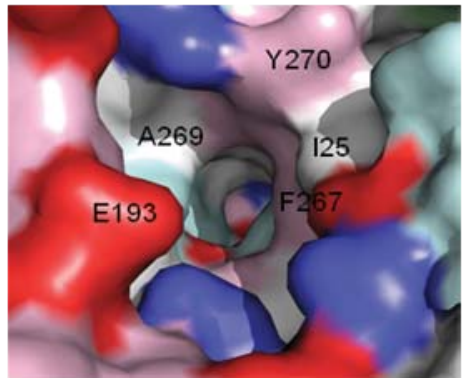

VLX-E

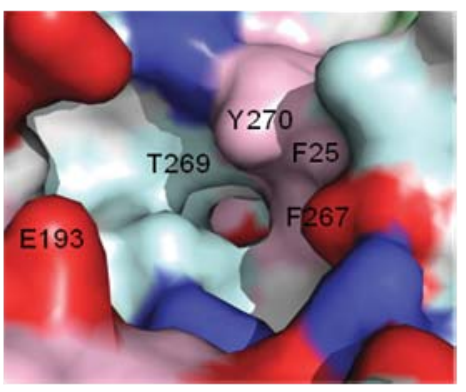

VLX-C

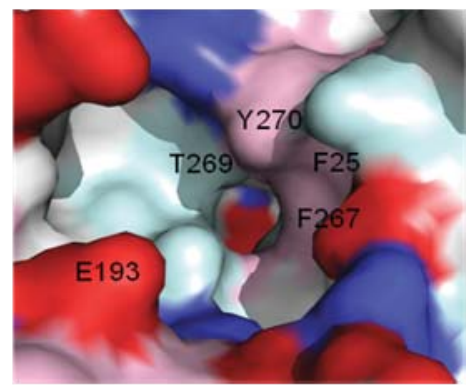

VLX-D

Figure 9. The molecular surface of the entry site: Red is for negative residues (Asp, Glu), blue for positive residues (Lys and Arg), white for the hydrophobic residues (Ala, Gly, Ile, Leu, Met, Pro, Val), yellow for semipolar (Cys), cyan for polar (His, Asn, Gln, Ser and Thr) and magenta for aromatic (Phe, Trp and Tyr). For the clarity of this discussion, the numbering system of VLX-B is being used. This figure was generated with the program PyMOL (v

$0.99)$.



Figure S1

Figure S1. Purified VLX isoforms. $1 \mu \mathrm{g}$ purified LOX loaded in each well 